\title{
New Results for Finite-Time Stability of Discrete-Time Linear Systems with Interval Time-Varying Delay
}

\author{
Sreten B. Stojanovic \\ Department of Engineering Sciences and Mathematics, Faculty of Technology, University of Niš, \\ Bulevar Oslobodjenja 124, 16000 Leskovac, Serbia \\ Correspondence should be addressed to Sreten B. Stojanovic; sstojanovic@tf.ni.ac.rs
}

Received 12 February 2015; Revised 6 April 2015; Accepted 8 April 2015

Academic Editor: Victor S. Kozyakin

Copyright (C) 2015 Sreten B. Stojanovic. This is an open access article distributed under the Creative Commons Attribution License, which permits unrestricted use, distribution, and reproduction in any medium, provided the original work is properly cited.

The problem of finite-time stability for linear discrete time systems with state time-varying delay is considered in this paper. Two finite sum inequalities for estimating weighted norms of delayed states are proposed in order to obtain less conservative stability criteria. By using Lyapunov-Krasovskii-like functional with power function, two sufficient conditions of finite-time stability are proposed and expressed in the form of linear matrix inequalities (LMIs), which are dependent on the minimum and maximum delay bounds. The numerical example is presented to illustrate the applicability of the developed results. It was shown that the obtained results are less conservative than some existing ones in the literature.

\section{Introduction}

Up to now, most of the existing literature related to stability systems is focused on Lyapunov asymptotic stability, which is defined over an infinite time interval. However, often, this stability concept is insufficient for practical purposes, because there are some real systems which require that the system states do not exceed the specified values over a fixed short time. A system could be Lyapunov stable but completely useless because it possesses undesirable transient performances, for instance, in the presence of saturation or controlling the trajectory of a space vehicle from an initial point to a final one in a prescribed time interval. Consequently, it is of particular interest to investigate the trajectories of the system only over a finite time interval. The described stability concept, based on the stability investigation in a limited time frame, is named finite-time stability (FTS). In that sense, the system is stable if the states of the system do not exceed the predefined boundaries on some fixed time interval. This stability concept was introduced in the era of modern control systems $[1,2]$, and it is still widely used nowadays as well. Initially, the concept had an academic value, and its practical applications were applied later on. With the development of the linear inequalities, the stability conditions that could be used for practical purposes were developed for both continuous [3-5] and discrete-time systems [6-8].
It should be noted that, in the existing literature, parallel to the previously defined FTS, there is another stability concept denoted by FTS, but with a different meaning. The second FTS concept is used to describe system whose states approach equilibrium point in a finite time [5]. In our paper, we consider the first concept of FTS.

Furthermore, FTS in the presence of exogenous input leads to the concept of finite-time boundedness (FTB) $[4,9$, 10] (a system is said to be FTB if, given a bound on the initial condition and characterization of the set of admissible inputs, the state variables remain below the prescribed limit for all inputs in the set).

In some practical systems (such as biological systems, mechanical systems, and networked control systems), time delay is inevitable. The existence of time delay might result in the performance deterioration or even instability of the system. Frequently, the time delay is not constant but time varying. A large number of researchers were involved in the study of time-delay systems (see, e.g., [11-13] and the references therein).

Similar to regular systems, the FTS concept can be applied to the class of time-delay systems. However, up to date, there are few works dealing with the FTS of time-delay systems. Some early results of FTS for time-delay systems can be found in [14]. The results of these investigations are 
conservative because they are based on the application of simple algebraic inequalities. Recently, using the theory of linear matrix inequality (LMI), more practical and precise results are obtained for continuous and discrete-time-delay systems.

In [15], the finite-time control problem for networked control systems with time-varying delays is investigated and a finite-time controller via state feedback is proposed to guarantee FTB. In $[16,17]$, the finite-time stability and stabilization for a class of linear continuous-time systems with constant time delay are studied. By using LyapunovKrasovskii-like (LKL) functional, some sufficient conditions that ensure finite-time performance of the considered systems are derived in the form of LMIs. The robust FTS is discussed in [17]. The controller design problem is solved by using the LMIs and the cone complementarity linearization iterative algorithm. In order to get less conservative criteria, a new form of LKL functional with power weighting function is introduced in [18]. In the last few years, with the great development of the switched system theory, the FTS and FTB problems of switched systems with time delay have received increasing attention (see, e.g., [19, 20] and the references therein). Recently, there has been rapidly growing interest in the problem of FTS analysis and synthesis of discrete-time systems with time-varying delay. In [21], the sufficient delaydependent FTS conditions are derived by using new LKL functional and an appropriate model transformation of the original system. A less conservative criterion is proposed in [22] by choosing a new LKL functional with power weighting function. In order to get less conservative criteria in [23], the original time-delay system is firstly transformed into two interconnected subsystems. Then, new sufficient conditions of FTS and stabilization are derived by using a two-term approximation of the time-varying delay.

The main purpose of this paper is to obtain less conservative stability criteria for finite-time stability for discretetime linear system with time-varying delay. Firstly, two finite sum inequalities for estimating weighted norms of delayed states are proposed. By using LKL functional with power function and finite sum inequalities, sufficient conditions of finite-time stability are proposed and expressed in the form of linear matrix inequalities (LMIs), which are dependent on the minimum and maximum delay bounds. Finally, a numerical example is given to illustrate the applicability of the developed results and the comparisons with some previous ones. It was shown that the obtained results are less conservative than some existing ones in the literature.

The rest of this paper is organized as follows. Section 2 gives the description of the considered system firstly and some necessary lemmas and remarks are listed. In Section 3, on the basis of the proposed finite sum inequalities and LKL functional, sufficient conditions are presented to guarantee the finite-time stability of the considered system. Section 4 gives one numerical example to illustrate the applicability of the developed results. Section 5 shows the summary of this paper.

Notations. The matrix transposition was denoted by a superscript “ $T$." $\mathfrak{R}^{n}$ and $\mathfrak{R}^{n \times m}$ are the $n$-dimensional Euclidean spaces and the set of all real matrices having dimension $n \times m$, respectively. $X>0$ denotes a real positive definite matrix, while $X>Y$ implies that the matrix $X-Y$ is a positive definite matrix. $\lambda_{\max }(X)\left(\lambda_{\min }(X)\right)$ denotes the maximum (minimum) of eigenvalues of a real symmetric matrix $X$.

\section{Preliminaries and Problem Formulation}

Consider the following discrete time system with timevarying delay:

$$
x(k+1)=A x(k)+A_{d} x(k-d(k)),
$$

where $x(k) \in \mathfrak{R}^{n}$ is the state vector and $A \in \mathfrak{R}^{n \times n}$ and $A_{d} \in \mathfrak{R}^{n \times n}$ are known constant matrices. The time delay $d(k)$ is assumed to be time varying and has lower $d_{m}$ and upper $d_{M}$ bounds such that

$$
0<d_{m} \leq d(k) \leq d_{M}
$$

The initial condition $\phi(\theta)$ is defined as

$$
x(\theta)=\phi(\theta), \quad \theta \in\left\{-d_{M},-d_{M}+1, \ldots,-1,0\right\}
$$

with

$$
\begin{aligned}
& \sup _{\theta \in\left\{-d_{M},-d_{M}+1, \ldots,-1\right\}}(\phi(\theta+1)-\phi(\theta))^{T}(\phi(\theta+1)-\phi(\theta)) \\
& \quad \leq \delta .
\end{aligned}
$$

In this paper, the purpose is to derive sufficient conditions which guarantee the finite-time stability of discrete-time system with time-varying delay (1). To study the finite-time stability, the following definition is necessarily introduced.

Definition 1. Discrete-time system with time-varying delay (1) is said to be finite-time stable with respect to $(\alpha, \beta, N)$, where $0 \leq \alpha<\beta$, if $\forall k \in\{1,2, \ldots, N\}$

$$
\sup _{j \in\left\{-d_{M},-d_{M}+1, \ldots, 0\right\}} \phi^{T}(j) \phi(j) \leq \alpha \Longrightarrow x^{T}(k) x(k)<\beta .
$$

The following lemmas will be used in the derivation of the main results.

Lemma 2. For any appropriately dimensioned matrices $Z=$ $Z^{T}>0, M$ and positive integers $d_{1}, d_{2}>d_{1}$, and $\gamma$, the inequality

$$
\begin{aligned}
& -\sum_{j=k-d_{2}}^{k-d_{1}-1} \gamma^{k-j} y^{T}(j) Z y(j) \\
& \leq \xi^{T}(k) c M Z^{-1} M^{T} \xi(k) \\
& \quad+2 \xi^{T}(k) M\left(x\left(k-d_{1}\right)-x\left(k-d_{2}\right)\right)
\end{aligned}
$$

holds, where $y(k)=x(k+1)-x(k), \xi(k)=$ $\left[\begin{array}{llll}x^{T}(k) & x^{T}(k-d(k)) & x^{T}\left(k-d_{1}\right) & x^{T}\left(k-d_{2}\right)\end{array}\right]^{T}$, and

$$
c=\frac{\gamma^{-d_{1}}-\gamma^{-d_{2}}}{\gamma-1} .
$$


Proof. Using the inequality

$$
\begin{aligned}
0 \geq & -\sum_{j=k-d_{2}}^{k-d_{1}-1}\left(M^{T} \xi(k)+\gamma^{k-j} Z y(j)\right)^{T}\left(\gamma^{k-j} Z\right)^{-1} \\
& \cdot\left(M^{T} \xi(k)+\gamma^{k-j} Z y(j)\right)
\end{aligned}
$$

we obtain

$$
\begin{aligned}
0 \geq & -\sum_{j=k-d_{2}}^{k-d_{1}-1} \xi^{T}(k) M\left(\gamma^{k-j} Z\right)^{-1} M^{T} \xi(k) \\
& -\sum_{j=k-d_{2}}^{k-d_{1}-1} 2 \xi^{T}(k) M\left(\gamma^{k-j} Z\right)^{-1} \gamma^{k-j} Z y(j) \\
& -\sum_{j=k-d_{2}}^{k-d_{1}-1} \gamma^{k-j} y^{T}(j) Z\left(\gamma^{k-j} Z\right)^{-1} \gamma^{k-j} Z y(j) \\
= & -\xi^{T}(k) M Z^{-1} M^{T} \xi(k) \sum_{j=k-d_{2}}^{k-d_{1}-1} \gamma^{k-j} \\
& -2 \xi^{T}(k) M \sum_{j=k-d_{2}}^{k-d_{1}-1} y(j)-\sum_{j=k-d_{2}}^{k-d_{1}-1} \gamma^{k-j} y^{T}(j) Z y(j)
\end{aligned}
$$

which implies

$$
\begin{aligned}
& -\sum_{j=k-d_{2}}^{k-d_{1}-1} \gamma^{k-j} y^{T}(j) Z y(j) \\
& \leq \xi^{T}(k) M Z^{-1} M^{T} \xi(k) \sum_{j=k-d_{2}}^{k-d_{1}-1} \gamma^{k-j} \\
& \quad+2 \xi^{T}(k) M \sum_{j=k-d_{2}}^{k-d_{1}-1} y(j) \\
& =\xi^{T}(k) c M Z^{-1} M^{T} \xi(k) \\
& \quad+2 \xi^{T}(k) M\left(x\left(k-d_{1}\right)-x\left(k-d_{2}\right)\right) .
\end{aligned}
$$

This completes the proof.

Lemma 3. For any appropriately dimensioned matrices $Z=$ $Z^{T}>0, X, Y$ and positive integers $d_{1}, d_{2}>d_{1}$, and $\gamma$, the inequality

$$
\begin{aligned}
& -\sum_{j=k-d_{2}}^{k-d_{1}-1} \gamma^{k-j} y^{T}(j) Z y(j) \\
& \leq \xi^{T}(k) c X \xi(k) \\
& \quad+2 \xi^{T}(k) Y\left(x\left(k-d_{1}\right)-x\left(k-d_{2}\right)\right)
\end{aligned}
$$

holds, where $y(k)=x(k+1)-x(k), \xi(k)=$ $\left[\begin{array}{llll}x^{T}(k) & x^{T}(k-d(k)) & x^{T}\left(k-d_{1}\right) & x^{T}\left(k-d_{2}\right)\end{array}\right]^{T}$, and

$$
\begin{aligned}
{\left[\begin{array}{ll}
X & Y \\
* & Z
\end{array}\right] } & \geq 0, \\
c & =\frac{\gamma^{-d_{1}}-\gamma^{-d_{2}}}{\gamma-1} .
\end{aligned}
$$

Proof. From (12), we have

$$
\left[\begin{array}{l}
a \\
b
\end{array}\right]^{T}\left[\begin{array}{ll}
X & Y \\
* & Z
\end{array}\right]\left[\begin{array}{l}
a \\
b
\end{array}\right] \geq 0 .
$$

Let $a=\gamma^{(k-j) / 2} \xi(k), b=\gamma^{(k-j) / 2} y(j)$. Then

$$
\begin{aligned}
& -\gamma^{-(k-j) / 2} \xi^{T}(k) \gamma^{-(k-j) / 2} X \xi(k) \\
& \leq \gamma^{(k-j) / 2} y^{T}(j) Z \gamma^{(k-j) / 2} y(j) \\
& \quad+2 \gamma^{-(k-j) / 2} \xi^{T}(k) Y \gamma^{(k-j) / 2} y(j), \\
& -\gamma^{-(k-j)} \xi^{T}(k) X \xi(k) \\
& \leq \gamma^{k-j} y^{T}(j) Z y(j)+2 \xi^{T}(k) Y y(j) .
\end{aligned}
$$

Summarizing the previous expression per $j$, from $j=k-d_{2}$ to $j=k-d_{1}-1$, we get

$$
\begin{aligned}
& -\sum_{j=k-d_{2}}^{k-d_{1}-1} \gamma^{-(k-j)} \xi^{T}(k) X \xi(k) \\
& \leq \sum_{j=k-d_{2}}^{k-d_{1}-1} \gamma^{k-j} y^{T}(j) Z y(j)+2 \xi^{T}(k) Y \sum_{j=k-d_{2}}^{k-d_{1}-1} y(j) \\
& =\sum_{j=k-d_{2}}^{k-d_{1}-1} \gamma^{k-j} y^{T}(j) Z y(j) \\
& \quad+2 \xi^{T}(k) Y\left(x\left(k-d_{1}\right)-x\left(k-d_{2}\right)\right) .
\end{aligned}
$$

Rearranging the above equation yields

$$
\begin{aligned}
& -\sum_{j=k-d_{2}}^{k-d_{1}-1} \gamma^{k-j} y^{T}(j) Z y(j) \\
& \leq \sum_{j=k-d_{2}}^{k-d_{1}-1} \gamma^{-(k-j)} \xi^{T}(k) X \xi(k) \\
& \quad+2 \xi^{T}(k) Y\left(x\left(k-d_{1}\right)-x\left(k-d_{2}\right)\right) \\
& =c \xi^{T}(k) X \xi(k) \\
& \quad+2 \xi^{T}(k) Y\left(x\left(k-d_{1}\right)-x\left(k-d_{2}\right)\right) .
\end{aligned}
$$

This completes the proof. 
Remark 4. Let $Y=M$. Then, the right sides of inequalities (6) and (11) become

$$
\begin{aligned}
& \xi^{T}(k) c M Z^{-1} M^{T} \xi(k) \\
& \quad+2 \xi^{T}(k) M\left(x\left(k-d_{1}\right)-x\left(k-d_{2}\right)\right), \\
& \xi^{T}(k) c X \xi(k)+2 \xi^{T}(k) M\left(x\left(k-d_{1}\right)-x\left(k-d_{2}\right)\right),
\end{aligned}
$$

respectively. From (12), for $Z>0$, the following inequality holds:

$$
X>M Z^{-1} M^{T}
$$

which implies

$$
\xi^{T}(k) c X \xi(k)>\xi^{T}(k) c M Z^{-1} M^{T} \xi(k) .
$$

Based on (18) and (20), we have

$$
\begin{aligned}
& \xi^{T}(k) c M Z^{-1} M^{T} \xi(k) \\
& \quad+2 \xi^{T}(k) M\left(x\left(k-d_{1}\right)-x\left(k-d_{2}\right)\right) \\
& <\xi^{T}(k) c X \xi(k) \\
& \quad+2 \xi^{T}(k) M\left(x\left(k-d_{1}\right)-x\left(k-d_{2}\right)\right) .
\end{aligned}
$$

Hence, it can be concluded that inequality (6) is less restrictive compared to inequality (11) if $Y=M$.

Remark 5. If we adopt $X=M Z^{-1} M^{T}$ and $Y=M$, then the right sides of inequalities (6) and (11) are equal to each other and

$$
\begin{aligned}
{\left[\begin{array}{ll}
X & Y \\
* & Z
\end{array}\right] } & =\left[\begin{array}{cc}
M Z^{-1} M^{T} & M \\
* & Z
\end{array}\right] \\
& =\left[\begin{array}{cc}
Z^{-1 / 2} M^{T} & Z^{1 / 2} \\
0 & 0
\end{array}\right]^{T}\left[\begin{array}{cc}
Z^{-1 / 2} M^{T} & Z^{1 / 2} \\
0 & 0
\end{array}\right] \\
& \geq 0 .
\end{aligned}
$$

Then, Lemma 2 can be considered as a particular case of Lemma 3.

\section{Main Results}

In the sequel, we will present two finite-time stability criteria for system (1).

Theorem 6. The discrete-time system (1) with time-varying delay is finite-time stable with respect to $\{\alpha, \beta, N\}$ if there exist a scalar $\gamma>1$, positive scalars $\lambda_{1}, \lambda_{2}, \lambda_{3}, \lambda_{4}, \lambda_{5}, \lambda_{6}, \lambda_{7}$, and $\lambda_{8}$, positive definite symmetric matrices $P, Q_{1}, Q_{2}, R_{1}$, and $R_{2}$, and matrices $L=\left[\begin{array}{llll}L_{1}^{T} & L_{2}^{T} & L_{3}^{T} & L_{4}^{T}\end{array}\right]^{T}, S=\left[\begin{array}{llll}S_{1}^{T} & S_{2}^{T} & S_{3}^{T} & S_{4}^{T}\end{array}\right]^{T}$, and $T=\left[\begin{array}{llll}T_{1}^{T} & T_{2}^{T} & T_{3}^{T} & T_{4}^{T}\end{array}\right]^{T}$, such that the following inequalities hold:

$$
\begin{aligned}
& {\left[\begin{array}{cccc}
\Sigma_{1}+\Sigma_{2} & c_{1} L & c_{1} S & c_{2} T \\
* & -c_{1} R_{1} & 0 & 0 \\
* & * & -c_{1} R_{1} & 0 \\
* & * & * & -c_{2} R_{2}
\end{array}\right]<0,} \\
& \lambda_{1} I<P<\lambda_{2} I, \\
& \lambda_{3} I<Q_{1}<\lambda_{4} I, \\
& \lambda_{5} I<Q_{2}<\lambda_{6} I, \\
& R_{1}<\lambda_{7} I, \\
& R_{2}<\lambda_{8} I, \\
& \gamma^{N} \alpha\left[\lambda_{2}+\mu_{1} \lambda_{4}+\mu_{2} \lambda_{6}\right]+\gamma^{N} \delta\left[\mu_{3} \lambda_{7}+\mu_{4} \lambda_{8}\right] \\
& \quad-\beta\left[\lambda_{1}+\mu_{1} \lambda_{3}+\mu_{2} \lambda_{5}\right]<0,
\end{aligned}
$$

where

$$
\begin{aligned}
\Sigma_{1} & =\left[\begin{array}{cccc}
\Omega_{11} & \Omega_{12} & 0 & 0 \\
* & \Omega_{22} & 0 & 0 \\
* & * & -\gamma^{d_{m}} Q_{2} & 0 \\
* & * & * & -\gamma^{d_{M}} Q_{1}
\end{array}\right], \\
\Omega_{11} & =A^{T} P A-\gamma P+Q_{1}+Q_{2}+\left(d_{M}-d_{m}\right)(A-I)^{T} R_{1}(A-I)+d_{m}(A-I)^{T} R_{2}(A-I), \\
\Omega_{12} & =A^{T} P A_{d}+\left(d_{M}-d_{m}\right)(A-I)^{T} R_{1} A_{d}+d_{m}(A-I)^{T} R_{2} A_{d}, \\
\Omega_{22} & =A_{d}^{T} P A_{d}+\left(d_{M}-d_{m}\right) A_{d}^{T} R_{1} A_{d}+d_{m} A_{d}^{T} R_{2} A_{d},
\end{aligned}
$$




$$
\begin{aligned}
& \Sigma_{2}=\left[\begin{array}{cccc}
T_{1}+T_{1}^{T} & L_{1}-S_{1}+T_{2}^{T} & S_{1}-T_{1}+T_{3}^{T} & -L_{1}+T_{4}^{T} \\
* & L_{2}+L_{2}^{T}-S_{2}-S_{2}^{T} & L_{3}^{T}+S_{2}-S_{3}^{T}-T_{2} & -L_{2}+L_{4}^{T}-S_{4}^{T} \\
* & * & S_{3}+S_{3}^{T}-T_{3}-T_{3}^{T} & -L_{3}+S_{4}^{T}-T_{4}^{T} \\
* & * & * & -L_{4}-L_{4}^{T}
\end{array}\right], \\
& c_{1}=\frac{\gamma^{-d_{m}-\gamma^{-d_{M}}}}{\gamma-1}, \\
& c_{2}=\frac{1-\gamma^{-d_{m}}}{\gamma-1}, \\
& \mu_{1}=\frac{\gamma^{d_{M}-1}}{\gamma-1}, \\
& \mu_{2}=\frac{\gamma^{d_{m}-1}}{\gamma-1}, \\
& \mu_{3}=\frac{\gamma^{d_{M}+1}-\gamma^{d_{m}+1}-(\gamma-1)\left(d_{M}-d_{m}\right)}{(\gamma-1)^{2}} \\
& \mu=\frac{\gamma(\gamma-1)^{2}}{\left.\gamma^{d_{m}}-1\right)-(\gamma-1) d_{m}} .
\end{aligned}
$$

Proof. Let us consider the following discrete LKL functional:

$$
V(x(k))=V_{1}(x(k))+V_{2}(x(k))+V_{3}(x(k)),
$$

where

$$
\begin{aligned}
V_{1}(x(k))= & x^{T}(k) P x(k), \\
V_{2}(x(k))= & \sum_{j=k-d_{M}}^{k-1} \gamma^{k-1-j} x^{T}(j) Q_{1} x(j) \\
& +\sum_{j=k-d m}^{k-1} \gamma^{k-1-j} x^{T}(j) Q_{2} x(j), \\
V_{3}(x(k))= & \sum_{j=-d_{M}} \sum_{\theta=k+j}^{k-1} \gamma^{k-1-\theta} y^{T}(\theta) R_{1} y(\theta) \\
& +\sum_{j=-d_{m}}^{-1} \sum_{\theta=k+j}^{k-1} \gamma^{k-1-\theta} y^{T}(\theta) R_{2} y(\theta) .
\end{aligned}
$$

Define $\Delta V(x(k))=V(x(k+1))-(x(k))$. Then, along with solution of system (1), we have

$$
\begin{aligned}
& \Delta V_{1}(x(k))=x^{T}(k+1) P x(k+1)-x^{T}(k) P x(k) \\
& =\left(A x(k)+A_{d} x(k-d(k))\right)^{T} P\left(A x(k)+A_{d} x(k\right. \\
& \quad-d(k)))-x^{T}(k) P x(k)+\gamma x^{T}(k) P x(k)
\end{aligned}
$$

$$
\begin{aligned}
& -\gamma x^{T}(k) P x(k)=x^{T}(k)\left(A^{T} P A-\gamma P\right) x(k) \\
& +2 x^{T}(k) A^{T} P A_{d} x(k-d(k))+x^{T}(k-d(k)) \\
& \cdot A_{d}^{T} P A_{d} x(k-d(k))+(\gamma-1) V_{1},
\end{aligned}
$$$$
\Delta V_{2}(x(k))=\sum_{j=k+1-d_{M}}^{k} \gamma^{k-j} x^{T}(j) Q_{1} x(j)
$$$$
-\sum_{j=k-d_{M}}^{k-1} \gamma^{k-1-j} x^{T}(j) Q_{1} x(j)+\sum_{j=k+1-d_{m}}^{k} \gamma^{k-j} x^{T}(j)
$$$$
\cdot Q_{2} x(j)-\sum_{j=k-d_{m}}^{k-1} \gamma^{k-1-j} x^{T}(j) Q_{2} x(j)
$$$$
=\gamma \sum_{j=k-d_{M}}^{k-1} \gamma^{k-1-j} x^{T}(j) Q_{1} x(j)+x^{T}(k) Q_{1} x(k)
$$$$
-\gamma^{d_{M}} x^{T}\left(k-d_{M}\right) Q_{1} x\left(k-d_{M}\right)
$$$$
-\sum_{j=k-d_{M}}^{k-1} \gamma^{k-1-j} x^{T}(j) Q_{1} x(j)
$$$$
+\gamma \sum_{j=k-d_{m}}^{k-1} \gamma^{k-1-j} x^{T}(j) Q_{2} x(j)+x^{T}(k) Q_{2} x(k)
$$$$
-\gamma^{d_{m}} x^{T}\left(k-d_{m}\right) Q_{2} x\left(k-d_{m}\right)
$$ 


$$
\begin{aligned}
& -\sum_{j=k-d_{m}}^{k-1} \gamma^{k-1-j} x^{T}(j) Q_{2} x(j)=x^{T}(k)\left(Q_{1}+Q_{2}\right) \\
& \text { - } x(k)-\gamma^{d_{M}} x^{T}\left(k-d_{M}\right) Q_{1} x\left(k-d_{M}\right) \\
& -\gamma^{d_{m}} x^{T}\left(k-d_{m}\right) Q_{2} x\left(k-d_{m}\right)+(\gamma-1) \\
& \text { - } V_{2}(x(k)) \text {, } \\
& \Delta V_{3}(x(k))=\sum_{j=-d_{M}}^{d_{m}-1} \sum_{\theta=k+1+j}^{k} \gamma^{k-\theta} y^{T}(\theta) R_{1} y(\theta) \\
& -\sum_{j=-d_{M}}^{d_{m}-1} \sum_{\theta=k+j}^{k-1} \gamma^{k-1-\theta} y^{T}(\theta) R_{1} y(\theta) \\
& +\sum_{j=-d_{m}}^{-1} \sum_{\theta=k+1+j}^{k} \gamma^{k-\theta} y^{T}(\theta) R_{2} y(\theta) \\
& -\sum_{j=-d_{m}}^{-1} \sum_{\theta=k+j}^{k-1} \gamma^{k-1-\theta} y^{T}(\theta) R_{2} y(\theta) \\
& =\sum_{j=-d_{M}}^{d_{m}-1}\left[\gamma \sum_{\theta=k+j}^{k-1} \gamma^{k-1-\theta} y^{T}(\theta) R_{1} y(\theta)\right. \\
& \left.+y^{T}(k) R_{1} y(k)-\gamma^{-j} y^{T}(k+j) R_{1} y(k+j)\right] \\
& +\sum_{j=-d_{m}}^{-1}\left[\gamma \sum_{\theta=k+j}^{k-1} \gamma^{k-1-\theta} y^{T}(\theta) R_{2} y(\theta)\right. \\
& \left.+y^{T}(k) R_{2} y(k)-\gamma^{-j} y^{T}(k+j) R_{2} y(k+j)\right] \\
& -\sum_{j=-d_{M}}^{d_{m}-1} \sum_{\theta=k+j}^{k-1} \gamma^{k-1-\theta} y^{T}(\theta) R_{1} y(\theta) \\
& -\sum_{j=-d_{m}}^{-1} \sum_{\theta=k+j}^{k-1} \gamma^{k-1-\theta} y^{T}(\theta) R_{2} y(\theta) \\
& \Delta V_{3}(x(k))=(\gamma-1) \sum_{j=-d_{M}}^{d_{m}-1} \sum_{\theta=k+j}^{k-1} \gamma^{k-1-\theta} y^{T}(\theta) R_{1} y(\theta) \\
& +(\gamma-1) \sum_{j=-d_{m}}^{-1} \sum_{\theta=k+j}^{k-1} \gamma^{k-1-\theta} y^{T}(\theta) R_{2} y(\theta) \\
& +\sum_{j=-d_{M}}^{d_{m}-1}\left[y^{T}(k) R_{1} y(k)\right. \\
& \left.-\gamma^{-j} y^{T}(k+j) R_{1} y(k+j)\right] \\
& +\sum_{j=-d_{m}}^{-1}\left[y^{T}(k) R_{2} y(k)\right.
\end{aligned}
$$$$
\begin{aligned}
& \left.-\gamma^{-j} y^{T}(k+j) R_{2} y(k+j)\right]=\left(d_{M}-d_{m}\right) y^{T}(k) \\
& \cdot R_{1} y(k)-\sum_{j=-d_{M}}^{d_{m}-1} \gamma^{-j} y^{T}(k+j) R_{1} y(k+j) \\
& +d_{m} y^{T}(k) R_{2} y(k)-\sum_{j=-d_{m}}^{-1} \gamma^{-j} y^{T}(k+j) R_{2} y(k \\
& +j)+(\gamma-1) V_{3}(x(k))=\left((A-I) x(k)+A_{d} x(k\right. \\
& -d(k)))^{T}\left(\left(d_{M}-d_{m}\right) R_{1}+d_{m} R_{2}\right)((A-I) x(k) \\
& \left.+A_{d} x(k-d(k))\right)-\sum_{j=k-d_{M}}^{k-d_{m}-1} \gamma^{k-j} y^{T}(j) R_{1} y(j) \\
& -\sum_{j=k-d_{m}}^{k-1} \gamma^{k-j} y^{T}(j) R_{2} y(j)+(\gamma-1) V_{3}(x(k)) .
\end{aligned}
$$

Hence, it is concluded that

$$
\begin{aligned}
\Delta V(x(k))= & (\gamma-1) V(x(k))+x^{T}(k) \Omega_{11} x(k) \\
& +2 x^{T}(k) \Omega_{12} x(k-d(k)) \\
& +x(k-d(k)) \Omega_{22} x(k-d(k)) \\
& -\gamma^{d_{M}} x^{T}\left(k-d_{M}\right) Q_{1} x\left(k-d_{M}\right) \\
& -\gamma^{d_{m}} x^{T}\left(k-d_{m}\right) Q_{2} x\left(k-d_{m}\right) \\
& -\sum_{j=k-d_{M}}^{k-d_{m}-1} \gamma^{k-j} y^{T}(j) R_{1} y(j) \\
& -\sum_{j=k-d_{m}}^{k-1} \gamma^{k-j} y^{T}(j) R_{2} y(j) .
\end{aligned}
$$

According to Lemma 2, we have

$$
\begin{aligned}
& -\sum_{j=k-d_{M}}^{k-d_{m}-1} \gamma^{k-j} y^{T}(j) R_{1} y(j) \\
& =-\sum_{j=k-d_{M}}^{k-d(k)-1} \gamma^{k-j} y^{T}(j) R_{1} y(j) \\
& \quad-\sum_{j=k-d(k)}^{k-d_{m}-1} \gamma^{k-j} y^{T}(j) R_{1} y(j) \\
& \leq \xi^{T}(k) c_{1}^{\prime} L Z^{-1} L^{T} \xi(k) \\
& +2 \xi^{T}(k) L\left(x(k-d(k))-x\left(k-d_{M}\right)\right) \\
& +\xi^{T}(k) c_{1}^{\prime \prime} S Z^{-1} S^{T} \xi(k) \\
& +2 \xi^{T}(k) S\left(x\left(k-d_{m}\right)-x(k-d(k))\right)
\end{aligned}
$$




$$
\begin{aligned}
& \leq \xi^{T}(k) c_{1} L Z^{-1} L^{T} \xi(k) \\
& +2 \xi^{T}(k)\left[\begin{array}{llll}
0 & L & 0 & -L
\end{array}\right] \xi(k) \\
& +\xi^{T}(k) c_{1} S Z^{-1} S^{T} \xi(k) \\
& +2 \xi^{T}(k)\left[\begin{array}{llll}
0 & -S & S & 0
\end{array}\right] \xi(k) \\
& =\xi^{T}(k) c_{1} L Z^{-1} L^{T} \xi(k) \\
& +\xi^{T}(k)\left(\left[\begin{array}{llll}
0 & L & 0 & -L
\end{array}\right]+\left[\begin{array}{llll}
0 & L & 0 & -L
\end{array}\right]^{T}\right) \xi(k) \\
& +\xi^{T}(k) c_{1} S Z^{-1} S^{T} \xi(k) \\
& +\xi^{T}(k)\left(\left[\begin{array}{llll}
0 & -S & S & 0
\end{array}\right]+\left[\begin{array}{llll}
0 & -S & S & 0
\end{array}\right]^{T}\right) \xi(k), \\
& -\sum_{j=k-d_{m}}^{k-1} \gamma^{k-j} y^{T}(j) R_{2} y(j) \\
& \leq \xi^{T}(k) c_{2} T Z^{-1} T^{T} \xi(k) \\
& +2 \xi^{T}(k) T\left(x(k)-x\left(k-d_{m}\right)\right) \\
& =\xi^{T}(k) c_{2} T Z^{-1} T^{T} \xi(k) \\
& +2 \xi^{T}(k)\left[\begin{array}{llll}
T & 0 & -T & 0
\end{array}\right] \xi(k) \\
& =\xi^{T}(k) c_{2} T Z^{-1} T^{T} \xi(k) \\
& +\xi^{T}(k)\left(\left[\begin{array}{llll}
T & 0 & -T & 0
\end{array}\right]+\left[\begin{array}{cccc}
T & 0 & -T & 0
\end{array}\right]^{T}\right) \xi(k),
\end{aligned}
$$

where

$$
\begin{gathered}
c_{1}^{\prime}=\frac{\gamma^{-d(k)}-\gamma^{-d_{M}}}{\gamma-1} \leq \frac{\gamma^{-d_{m}}-\gamma^{-d_{M}}}{\gamma-1} \stackrel{\wedge}{=} c_{1}, \\
c_{1}^{\prime \prime}=\frac{\gamma^{-d_{m}}-\gamma^{-d(k)}}{\gamma-1} \leq \frac{\gamma^{-d_{m}}-\gamma^{-d_{M}}}{\gamma-1} \stackrel{\wedge}{=} c_{1} .
\end{gathered}
$$

Taking (30)-(31) into account, we obtain

$$
\begin{aligned}
& \Delta V(x(k))-(\gamma-1) V(x(k))=\xi^{T}(k) \\
& \quad \cdot\left(\Sigma_{1}+\Sigma_{2}+c_{1} L Z^{-1} L^{T}+c_{1} S Z^{-1} S^{T}+c_{2} T Z^{-1} T^{T}\right) \\
& \quad \cdot \xi(k) .
\end{aligned}
$$

If

$$
\Sigma_{1}+\Sigma_{2}+c_{1} L Z^{-1} L^{T}+c_{1} S Z^{-1} S^{T}+c_{2} T Z^{-1} T^{T}<0
$$
then

$$
\begin{aligned}
\Delta V & (x(k))-(\gamma-1) V(x(k)) \\
& =V(x(k+1))-V(x(k))-(\gamma-1) V(x(k)) \\
& =V(x(k+1))-\gamma V(x(k))<0 .
\end{aligned}
$$

Applying (35) iteratively, we obtain

$$
\begin{gathered}
V(x(k))<\gamma V(x(k-1))<\gamma^{2} V(x(k-2))<\cdots \\
<\gamma^{k} V(x(0)), \quad k=1,2,3, \ldots
\end{gathered}
$$

Based on the fact that $\gamma>1, \phi^{T}(j) \phi(j) \leq \alpha$, and (4), we get

$$
\begin{aligned}
& V(x(0))=x^{T}(0) P x(0)+\sum_{j=-d_{M}}^{-1} \gamma^{-1-j} x^{T}(j) Q_{1} x(j) \\
& +\sum_{j=-d_{m}}^{-1} \gamma^{-1-j} x^{T}(j) Q_{2} x(j) \\
& +\sum_{j=-d_{M}+1}^{d_{m}-1} \sum_{\theta=j}^{-1} \gamma^{-1-\theta} y^{T}(\theta) R_{1} y(\theta) \\
& +\sum_{j=-d_{m}}^{-1} \sum_{\theta=j}^{-1} \gamma^{-1-\theta} y^{T}(\theta) R_{2} y(\theta) \\
& \leq \alpha \lambda_{\text {max }}(P) \\
& +\lambda_{\max }\left(Q_{1}\right) \sum_{j=-d_{M}}^{-1} \gamma^{-1-j} x^{T}(j) x(j) \\
& +\lambda_{\max }\left(Q_{2}\right) \sum_{j=-d_{m}}^{-1} \gamma^{-1-j} x^{T}(j) x(j) \\
& +\lambda_{\max }\left(R_{1}\right) \sum_{j=-d_{M}+1}^{d_{m}-1} \sum_{\theta=j}^{-1} \gamma^{-1-\theta} y^{T}(\theta) y(\theta) \\
& +\lambda_{\max }\left(R_{2}\right) \sum_{j=-d_{m}}^{-1} \sum_{\theta=j}^{-1} \gamma^{-1-\theta} y^{T}(\theta) y(\theta) \\
& <\alpha \lambda_{\max }(P)+\alpha \lambda_{\max }\left(Q_{1}\right) \sum_{j=-d_{M}}^{-1} \gamma^{-1-j} \\
& +\alpha \lambda_{\max }\left(Q_{2}\right) \sum_{j=-d_{m}}^{-1} \gamma^{-1-j} \\
& +\delta \lambda_{\max }\left(R_{1}\right) \sum_{j=-d_{M}+1}^{d_{m}-1} \sum_{\theta=j}^{-1} \gamma^{-1-\theta} \\
& +\delta \lambda_{\max }\left(R_{2}\right) \sum_{j=-d_{m}}^{-1} \sum_{\theta=j}^{-1} \gamma^{-1-\theta}
\end{aligned}
$$

that is,

$$
\begin{aligned}
& V(x(0)) \\
& <\alpha\left[\lambda_{\max }(P)+\mu_{1} \lambda_{\max }\left(Q_{1}\right)+\mu_{2} \lambda_{\max }\left(Q_{2}\right)\right] \\
& \quad+\delta\left[\mu_{3} \lambda_{\max }\left(R_{1}\right)+\mu_{4} \lambda_{\max }\left(R_{2}\right)\right],
\end{aligned}
$$


where

$$
\begin{aligned}
& \mu_{1}=\sum_{j=-d_{M}}^{-1} \gamma^{-1-j}=\frac{\gamma^{d_{M}}-1}{\gamma-1}, \\
& \mu_{2}=\sum_{j=-d_{m}}^{-1} \gamma^{-1-j}=\frac{\gamma^{d_{m}}-1}{\gamma-1} \\
& \mu_{3}=\sum_{j=-d_{M}+1}^{d_{m}-1} \sum_{\theta=j}^{-1} \gamma^{-1-\theta} \\
& =\sum_{\theta=-d_{M}}^{-1} \gamma^{-1-\theta}+\sum_{\theta=-d_{M}+1}^{-1} \gamma^{-1-\theta}+\cdots+\sum_{\theta=-d_{m}-1}^{-1} \gamma^{-1-\theta} \\
& =\frac{\gamma^{d_{M}}-1}{\gamma-1}+\frac{\gamma^{d_{M}-1}-1}{\gamma-1}+\cdots+\frac{\gamma^{d_{m}+1}-1}{\gamma-1} \\
& =\frac{\gamma^{d_{M}+1}-\gamma^{d_{m}+1}-(\gamma-1)\left(d_{M}-d_{m}\right)}{(\gamma-1)^{2}}, \\
& \mu_{4}=\sum_{j=-d_{m}}^{-1} \sum_{\theta=j}^{-1} \gamma^{-1-\theta} \\
& =\sum_{\theta=-d_{m}}^{-1} \gamma^{-1-\theta}+\sum_{\theta=-d_{m}+1}^{-1} \gamma^{-1-\theta}+\cdots+\sum_{\theta=-1}^{-1} \gamma^{-1-\theta} \\
& =\frac{\gamma^{d_{m}}-1}{\gamma-1}+\frac{\gamma^{d_{m}-1}-1}{\gamma-1}+\cdots+\frac{\gamma^{1}-1}{\gamma-1} \\
& =\frac{\gamma\left(\gamma^{d_{m}}-1\right)-(\gamma-1) d_{m}}{(\gamma-1)^{2}} .
\end{aligned}
$$

Note that

$$
\begin{aligned}
V(x(k))> & \lambda_{\min }(P) x^{T}(k) x(k) \\
& +\lambda_{\min }\left(Q_{1}\right) \sum_{j=k-d_{M}}^{k-1} \gamma^{k-1-j} x^{T}(j) x(j) \\
& +\lambda_{\min }\left(Q_{2}\right) \sum_{j=k-d_{m}}^{k-1} \gamma^{k-1-j} x^{T}(j) x(j) .
\end{aligned}
$$

If the following condition is valid:

$$
\begin{aligned}
& \gamma^{k} V(x(0)) \\
& \quad<\beta\left[\lambda_{\min }(P)+\mu_{1} \lambda_{\min }\left(Q_{1}\right)+\mu_{2} \lambda_{\min }\left(Q_{2}\right)\right],
\end{aligned}
$$

then

$$
\begin{aligned}
& \lambda_{\min }(P) x^{T}(k) x(k) \\
& +\lambda_{\min }\left(Q_{1}\right) \sum_{j=k-d_{M}}^{k-1} \gamma^{k-1-j} x^{T}(j) x(j) \\
& +\lambda_{\min }\left(Q_{2}\right) \sum_{j=k-d_{m}}^{k-1} \gamma^{k-1-j} x^{T}(j) x(j)<V(x(k))
\end{aligned}
$$

$$
\begin{aligned}
& <\gamma^{k} V(x(0)) \\
& <\gamma^{k} \alpha\left[\lambda_{\max }(P)+\mu_{1} \lambda_{\max }\left(Q_{1}\right)+\mu_{2} \lambda_{\max }\left(Q_{2}\right)\right] \\
& +\gamma^{k} \delta\left[\mu_{3} \lambda_{\max }\left(R_{1}\right)+\mu_{4} \lambda_{\max }\left(R_{2}\right)\right] \\
& <\beta\left[\lambda_{\min }(P)+\mu_{1} \lambda_{\min }\left(Q_{1}\right)+\mu_{2} \lambda_{\text {min }}\left(Q_{2}\right)\right]
\end{aligned}
$$

which implies $x^{T}(k) x(k)<\beta$ for $k=1,2, \ldots, N$. Therefore, system (1) is finite-time stable.

By using inequalities (24) we have

$$
\begin{aligned}
& \gamma^{N} \alpha\left[\lambda_{2}+\mu_{1} \lambda_{4}+\mu_{2} \lambda_{6}\right]+\gamma^{N} \delta\left[\mu_{3} \lambda_{7}+\mu_{4} \lambda_{8}\right] \\
& \quad<\beta\left[\lambda_{1}+\mu_{1} \lambda_{3}+\mu_{2} \lambda_{5}\right]
\end{aligned}
$$

which is equivalent to (25). This completes the proof.

Theorem 7. The discrete-time system with time-varying delay (1) is finite-time stable with respect to $\{\alpha, \beta, N\}$ if there exist a scalar $\gamma>1$, positive scalars $\lambda_{1}, \lambda_{2}, \lambda_{3}, \lambda_{4}, \lambda_{5}, \lambda_{6}, \lambda_{7}$, and $\lambda_{8}$, positive definite symmetric matrices $P, Q_{1}, Q_{2}, R_{1}$, and $R_{2}$, and matrices $G, H, M, L=\left[\begin{array}{llll}L_{1}^{T} & L_{2}^{T} & L_{3}^{T} & L_{4}^{T}\end{array}\right]^{T}, S=$ $\left[\begin{array}{llll}S_{1}^{T} & S_{2}^{T} & S_{3}^{T} & S_{4}^{T}\end{array}\right]^{T}$, and $T=\left[\begin{array}{llll}T_{1}^{T} & T_{2}^{T} & T_{3}^{T} & T_{4}^{T}\end{array}\right]^{T}$, such that the following inequalities hold:

$$
\begin{aligned}
& \Sigma_{1}+\Sigma_{2}+c_{1}(G+H)+c_{2} M<0, \\
& {\left[\begin{array}{cc}
G & L \\
* & R_{1}
\end{array}\right] \geq 0,} \\
& {\left[\begin{array}{cc}
H & S \\
* & R_{1}
\end{array}\right] \geq 0,} \\
& {\left[\begin{array}{ll}
M & T \\
* & R_{2}
\end{array}\right] \geq 0,} \\
& \lambda_{1} I<P<\lambda_{2} I, \\
& \lambda_{3} I<Q_{1}<\lambda_{4} I, \\
& \lambda_{5} I<Q_{2}<\lambda_{6} I, \\
& R_{1}<\lambda_{7} I, \\
& R_{2}<\lambda_{8} I, \\
& \gamma^{N} \alpha\left[\lambda_{2}+\mu_{1} \lambda_{4}+\mu_{2} \lambda_{6}\right]+\gamma^{N} \delta\left[\mu_{3} \lambda_{7}+\mu_{4} \lambda_{8}\right] \\
& -\beta\left[\lambda_{1}+\mu_{1} \lambda_{3}+\mu_{2} \lambda_{5}\right]<0,
\end{aligned}
$$

where $\Sigma_{1}, \Sigma_{2}, c_{1}, c_{2}, \mu_{1}, \mu_{2}, \mu_{3}$, and $\mu_{4}$ are defined by (26). 
Proof. This proof is based on the proof of Theorem 6. Starting from expression (30) and using Lemma 3, we have

$$
\begin{aligned}
& -\sum_{j=k-d_{M}}^{k-d_{m}-1} \gamma^{k-j} y^{T}(j) R_{1} y(j) \\
& =-\sum_{j=k-d_{M}}^{k-d(k)-1} \gamma^{k-j} y^{T}(j) R_{1} y(j) \\
& -\sum_{j=k-d(k)}^{k-d_{m}-1} \gamma^{k-j} y^{T}(j) R_{1} y(j) \\
& \leq \xi^{T}(k) c_{1}^{\prime} G \xi(k) \\
& +2 \xi^{T}(k) L\left(x(k-d(k))-x\left(k-d_{M}\right)\right) \\
& +\xi^{T}(k) c_{1}^{\prime \prime} H \xi(k) \\
& +2 \xi^{T}(k) S\left(x\left(k-d_{m}\right)-x(k-d(k))\right) \\
& \leq \xi^{T}(k) c_{1} G \xi(k)+2 \xi^{T}(k)\left[\begin{array}{llll}
0 & L & 0 & -L
\end{array}\right] \xi(k) \\
& +\xi^{T}(k) c_{1} H \xi(k)+2 \xi^{T}(k)\left[\begin{array}{llll}
0 & -S & S & 0
\end{array}\right] \xi(k) \\
& =\xi^{T}(k) c_{1} G \xi(k) \\
& +\xi^{T}(k)\left(\left[\begin{array}{llll}
0 & L & 0 & -L
\end{array}\right]+\left[\begin{array}{llll}
0 & L & 0 & -L
\end{array}\right]^{T}\right) \xi(k) \\
& +\xi^{T}(k) c_{1} H \xi(k) \\
& +\xi^{T}(k)\left(\left[\begin{array}{llll}
0 & -S & S & 0
\end{array}\right]+\left[\begin{array}{llll}
0 & -S & S & 0
\end{array}\right]^{T}\right) \xi(k), \\
& -\sum_{j=k-d_{m}}^{k-1} \gamma^{k-j} y^{T}(j) R_{2} y(j) \\
& \leq \xi^{T}(k) c_{2} M \xi(k)+2 \xi^{T}(k) T\left(x(k)-x\left(k-d_{m}\right)\right) \\
& =\xi^{T}(k) c_{2} M \xi(k)+2 \xi^{T}(k)\left[\begin{array}{llll}
T & 0 & -T & 0
\end{array}\right] \xi(k) \\
& =\xi^{T}(k) c_{2} M \xi(k) \\
& +\xi^{T}(k)\left(\left[\begin{array}{llll}
T & 0 & -T & 0
\end{array}\right]+\left[\begin{array}{llll}
T & 0 & -T & 0
\end{array}\right]^{T}\right) \xi(k) .
\end{aligned}
$$

In addition, by combining (30) and inequalities (48), we can obtain

$$
\begin{aligned}
\Delta V & (x(k))-(\gamma-1) V(x(k)) \\
\quad & =\xi^{T}(k)\left(\Sigma_{1}+\Sigma_{2}+c_{1}(G+H)+c_{2} M\right) \xi(k) .
\end{aligned}
$$

Based on inequality (44), we get

$$
V(x(k+1))<\gamma V(x(k)) ;
$$

that is,

$$
V(x(k))<\gamma^{k} V(x(0)), \quad k=1,2,3, \ldots
$$

Further procedure is identical to the proof of Theorem 6.
Remark 8. In the finite-time stability theory, the main problem is how to obtain the inequality $V(x(k+1))<\gamma V(x(k))$ (see (35) and (50)) using a minimum of approximations. By introducing the power function $\gamma^{k-j}$ in LKL functional, the previous inequality can be obtained using only approximations of the following quadratic form, which was used in (6) and (11):

$$
-\sum_{j=k-d_{2}}^{k-d_{1}-1} \gamma^{k-j} y^{T}(j) Z y(j) .
$$

Therefore, it is important that the quadratic form should be approximated as precisely as possible, in order to reduce the conservatism of the FTS criterion. In this paper, this problem is solved by using Lemmas 2 and 3 .

In most of the existing literature (see, e.g., [21, 23] and the references therein), the power function is not used in the LKL functional. Consequently, various techniques for approximation have been used for obtaining the inequality $V(x(k+1))<\gamma V(x(k))$, which lead to increasing the conservatism of FTS criteria. On the other hand, the LKL functional in [22] is proposed with the power function, but the quadratic form with the power function is approximated using the following quadratic form without power function:

$$
\begin{aligned}
& -\sum_{j=k-d_{2}}^{k-d_{1}-1} \gamma^{k-j} y^{T}(j) Z y(j) \\
& \leq-\gamma^{d_{1}+1} \sum_{j=k-d_{2}}^{k-d_{1}-1} y^{T}(j) Z y(j),
\end{aligned}
$$

which introduces additional conservatism in FTS criteria. Thus, it can be expected that introducing the power function in LKL functional and using the less restrictive inequalities (6) and (11) lead to smaller conservatism of FTS criteria.

Remark 9. Inequality (12) is nonstrict LMIs. However, Lemma 3 also remains valid in the case of the following strict LMIs:

$$
\left[\begin{array}{ll}
X & Y \\
* & Z
\end{array}\right]>0
$$

but its result becomes somewhat restrictive. Accordingly, nonstrict LMIs in (45) can be replaced by the following restrictive strict LMIs:

$$
\begin{aligned}
& {\left[\begin{array}{ll}
G & L \\
* & R_{1}
\end{array}\right]>0,} \\
& {\left[\begin{array}{cc}
H & S \\
* & R_{1}
\end{array}\right]>0,} \\
& {\left[\begin{array}{ll}
M & T \\
* & R_{2}
\end{array}\right]>0,}
\end{aligned}
$$

which can be easily calculated using some strict LMI solvers, similar to LMI Toolbox in Matlab. Note that the nonstrict LMIs (55) are used in Numerical Example. 
Remark 10. Taking into account Remarks 4 and 9, it can be expected that the result of Theorem 7 will be somewhat restrictive compared to Theorem 6 . This claim will be demonstrated in Example 1.

Remark 11. In order to compare the results of this paper with those from the existing literature, the smallest eligible value of the parameter $\beta, \beta_{m}$, and the largest eligible value of time delay $d(k), d_{M m}$, are adopted as criteria. If the given criterion, compared to other criteria, has a higher (smaller) computed value of $\beta_{m}\left(d_{M m}\right)$, then its conservatism is greater. For the given criterion, the computed value of $\beta_{m}\left(d_{M m}\right)$ is always greater (smaller) than its theoretical value $\beta_{m}^{t}\left(d_{M m}^{t}\right)$, which represents the minimal (maximal) values of $\beta_{m}\left(d_{M m}\right)$ such that the considered system is FTS. The system simulation can be used for the estimation of the parameter $\beta_{m}^{t}\left(d_{M m}^{t}\right)$ (see Examples 1-3).

The algorithm for computing the smallest eligible value of the parameter $\beta_{m}$ is described by the following steps.

Algorithm 12. Consider the following steps.

Step 1. Adopt a value of computational precision $\Delta>0$ of the smallest eligible value of the parameter $\beta\left(\beta_{m}\right)$.

Step 2. Set $k=1$. Choose a sufficiently large value for initial value for $\beta_{0}$ such that LMIs (23)-(25) (Theorem 6) or (44)(47) (Theorem 7) are feasible. Select new value $\beta_{1} \in\left[\alpha, \beta_{0}\right]$.

Step 3. Set $k=2$. If there is a feasible solution of LMIs (23)-(25) (Theorem 6) or (44)-(47) (Theorem 7) for given $\beta_{k-1}$, then select new value $\beta_{k} \in\left[\alpha, \beta_{k-1}\right]$; otherwise, $\beta_{k} \in$ $\left[\beta_{k-1}, \beta_{k-2}\right]$.

Step 4. Set $k=k+1$. If there is a feasible solution of LMIs (23)-(25) (Theorem 6) or (44)-(47) (Theorem 7) for given $\beta_{k-1}$, then select new value $\beta_{k} \in\left[\beta_{k-2}, \beta_{k-1}\right]$; otherwise, $\beta_{k} \in\left[\beta_{k-1}, \beta_{k-2}\right]$.

Step 5. If $\left|\beta_{k}-\beta_{k 1}\right|<\Delta$, then $\beta_{m}=\beta_{k}$ and exit. Otherwise, go to Step 4 .

A similar algorithm can be proposed for computing the largest eligible value of time delay $d_{\mathrm{Mm}}$.

\section{Numerical Example}

The effectiveness of the results presented in the previous section is now shown by means of numerical examples. We investigate the finite-time stability by comparing the smallest eligible values of parameter $\beta_{m}$ or the largest eligible values of time delay $d_{M m}$.

Example 1. Consider the following discrete-time system with time-varying delay:

$$
x(k+1)=\left[\begin{array}{ccc}
0.2 & 0.1 & 0.1 \\
0.2 & 0.1 & 0.4 \\
0.2 & 1 & 0.2
\end{array}\right] x(k)
$$

$$
\begin{array}{r}
+\left[\begin{array}{ccc}
0.1 & 0.01 & 0.1 \\
0.1 & 0.01 & 0.1 \\
0.1 & 0.1 & 0.01
\end{array}\right] x(k-d(k)), \\
d_{m}=2, d_{M}=5 .
\end{array}
$$

The aim is to examine the FTS of system (56) with respect to $(3, \beta, N)$ for $N=5,10,20$ and find the smallest eligible value of the parameter $\beta_{m}$.

Applying Theorem 6 , for $\delta=1.1, N=10$, and $\gamma=1.14$, we get the following feasible solution:

$$
\begin{aligned}
& \beta_{m}=\beta_{m 1}=37, \\
& P=\left[\begin{array}{ccc}
7.2962 & -0.0953 & -0.4689 \\
* & 7.2793 & -0.7771 \\
* & * & 3.6941
\end{array}\right], \\
& Q_{1}=\left[\begin{array}{ccc}
0.7662 & -0.0008 & -0.0010 \\
* & 0.7498 & -0.0057 \\
* & * & 0.7463
\end{array}\right] \text {, } \\
& Q_{2}=\left[\begin{array}{ccc}
0.7811 & 0.0124 & 0.0816 \\
* & 0.2411 & -0.0673 \\
* & * & 0.2316
\end{array}\right] \\
& R_{1}=\left[\begin{array}{ccc}
2.4430 & -0.1324 & 0.3298 \\
* & 1.2104 & 1.0944 \\
* & * & 1.4342
\end{array}\right], \\
& R_{2}=\left[\begin{array}{ccc}
0.1691 & 0.0056 & -0.0337 \\
* & 0.1570 & 0.0918 \\
* & * & 0.0811
\end{array}\right], \\
& \lambda_{1}=3.4667 \text {, } \\
& \lambda_{2}=7.4649 \text {, } \\
& \lambda_{3}=0.7408 \text {, } \\
& \lambda_{4}=0.7709 \text {, } \\
& \lambda_{5}=0.1581 \text {, } \\
& \lambda_{6}=0.8030 \text {, } \\
& \lambda_{7}=2.6171 \text {, } \\
& \lambda_{8}=0.2496 \text {, }
\end{aligned}
$$




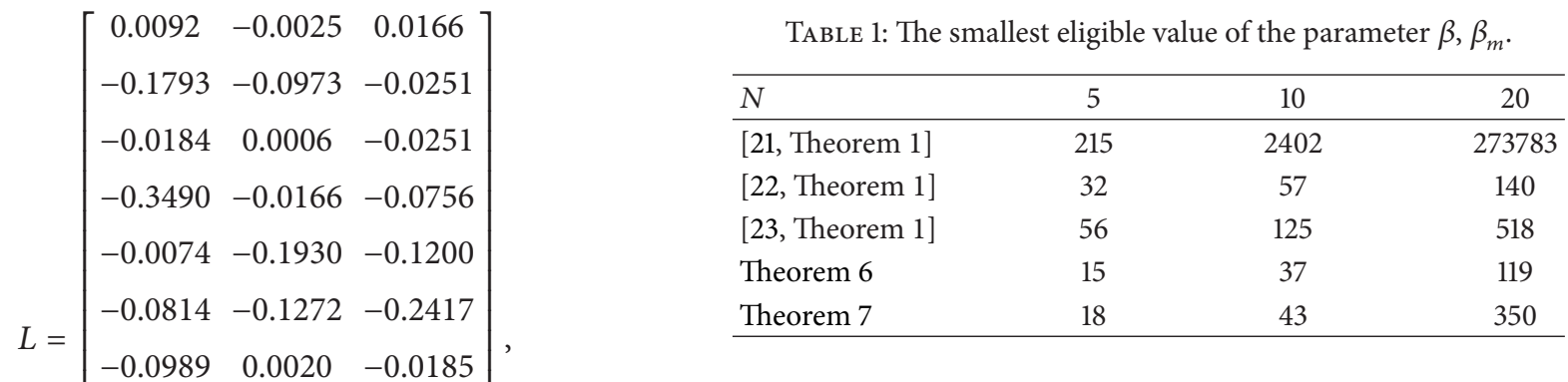

Further, the smallest eligible values of the parameter $\beta_{m}$, in this paper and the literature [21-23], are shown in Table 1 for $N=5,10$, and 20. In this paper, the values of $\beta_{m}$ are smaller than those in [21-23]. Thus, for this example, the approach in this paper is less conservative than those in [2123]. Theorem 7 shows a slightly greater conservatism than Theorem 6. The explanation for this is given in Remarks 4, 9 , and 10 .

In order to verify the stability properties, a computer simulation of the system with the random time-varying delay, $2 \leq d(k) \leq 5$, is performed under the conditions

$$
\begin{aligned}
\phi_{\theta \in\{-5,-4, \ldots, 0\}} & =\left[\begin{array}{lllll}
\phi(-5) & \cdots & \phi(0)
\end{array}\right] \\
& =\left[\begin{array}{llllll}
1 & 1 & 1 & 1 & 1 & 1 \\
1 & 1 & 1 & 1 & 1 & 1 \\
1 & 1 & 1 & 1 & 1 & 1
\end{array}\right]^{T} .
\end{aligned}
$$

It is obvious that

$$
\begin{aligned}
& \sup _{\theta \in\{-5,-4, \ldots, 0\}} \phi^{T}(\theta) \phi(\theta) \leq \alpha=3, \\
& \sup _{\theta \in\{-5,-4, \ldots,-1\}}(\phi(\theta+1)-\phi(\theta))^{T}(\phi(\theta+1)-\phi(\theta)) \\
& \leq \delta=1.1 .
\end{aligned}
$$

Figures 1-3 show the initial state responses of system (56), time-varying delay as random variable, and the norm of the initial state responses. Obviously, the considered system is not asymptotically stable. From Figure 3(b), the theoretical value of $\beta_{m}, \beta_{m}^{t}$, which is obtained by computer simulation amounts to $\beta_{m}^{t}=6.25$. As the conditions $\beta_{m 1}=37>\beta_{m}^{t}$ and $\beta_{m 2}=$ $43>\beta_{m}^{t}$ are satisfied, based on Theorems 6 and 7, we can conclude that system (56) is finite-time stable.

Example 2. Consider the following discrete-time system with time-varying delay:

$$
\begin{aligned}
& x(k+1)= {\left[\begin{array}{ll}
0.60 & 0.00 \\
0.35 & 0.70
\end{array}\right] x(k) } \\
&+\left[\begin{array}{ll}
0.10 & 0.00 \\
0.20 & 0.10
\end{array}\right] x(k-d(k)), \\
& \qquad d_{m}=2 \leq d(k) \leq d_{M},
\end{aligned}
$$

which has been considered in [21-23]. In addition, we choose $(\alpha, \beta, N)=(2.1,80,100)$. By using Theorem 6, for fixed 


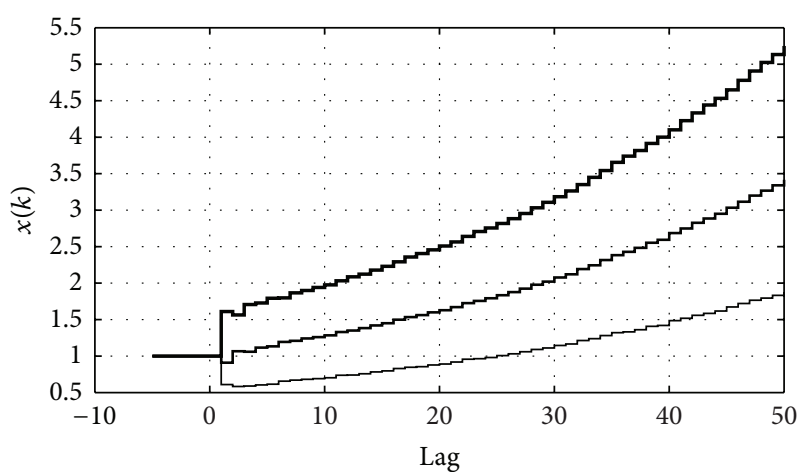

$$
\begin{aligned}
& -x_{1}(t) \\
& -\quad x_{2}(t) \\
& -x_{3}(t)
\end{aligned}
$$

FIGURE 1: The initial state responses of system (56).

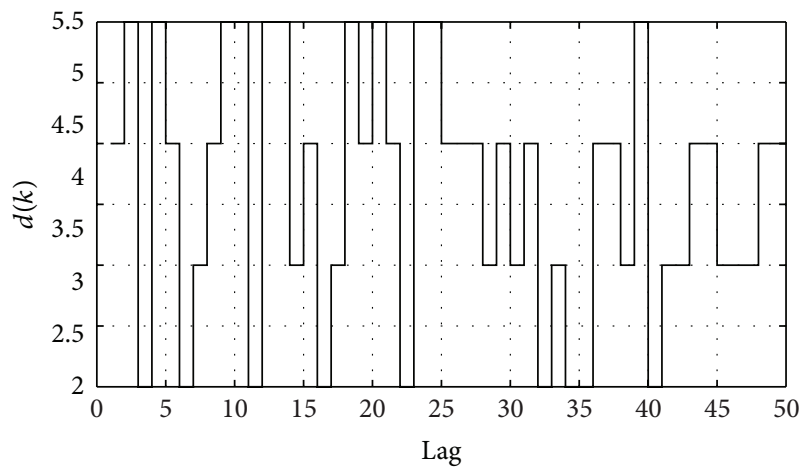

Figure 2: Time-varying delay $2 \leq d(k) \leq 5$.

$\gamma=1.000002$, the following feasible solution with the largest eligible value of time delay $d_{M m}$ is obtained:

$$
\begin{aligned}
d_{M m} & =10, \\
P & =\left[\begin{array}{cc}
4961.7 & 38.0 \\
38.0 & 306.8
\end{array}\right], \\
Q_{1} & =\left[\begin{array}{ll}
715.81 & -36.91 \\
-36.91 & 76.77
\end{array}\right], \\
Q_{2} & =\left[\begin{array}{ll}
1088.8 & -41.1 \\
-41.1 & 27.3
\end{array}\right], \\
R_{1} & =\left[\begin{array}{cc}
1031.4 & 109.0 \\
109.0 & 65.0
\end{array}\right], \\
R_{2} & =\left[\begin{array}{cc}
59.35 & -52.43 \\
-52.43 & 47.07
\end{array}\right], \\
\lambda_{1} & =306.4, \\
\lambda_{2} & =4963.9, \\
\lambda_{3} & =74.6,
\end{aligned}
$$

TABLE 2: The largest eligible value of time delay $d(k)\left(d_{M m}\right)$.

\begin{tabular}{lc}
\hline [21, Theorem 1] & 7 \\
{$[22$, Theorem 1] } & 10 \\
[23, Theorem 1] & 10 \\
Theorem 6 & 10 \\
\hline
\end{tabular}

$$
\begin{aligned}
& \lambda_{4}=718.1 \\
& \lambda_{5}=25.7, \\
& \lambda_{6}=1091.3, \\
& \lambda_{7}=1043.7, \\
& \lambda_{8}=107.2
\end{aligned}
$$$$
L=\left[\begin{array}{cc}
-0.04 & -0.02 \\
1.19 & 0.10 \\
-110.05 & -11.55 \\
-11.68 & -7.82 \\
-11.34 & -1.24 \\
-1.72 & -0.24 \\
27.21 & 2.94 \\
13.35 & 7.52
\end{array}\right] \text {, }
$$$$
S=\left[\begin{array}{cc}
-2.31 & -0.06 \\
0.68 & -0.05 \\
110.45 & 11.58 \\
12.09 & 7.86 \\
8.58 & 0.69 \\
-16.43 & -8.01 \\
11.40 & 1.27 \\
0.81 & 0.15
\end{array}\right] \text {, }
$$

$$
T=\left[\begin{array}{cc}
-23.59 & 20.82 \\
23.79 & -21.37 \\
2.23 & -1.96 \\
0.02 & -0.01 \\
-1.42 & 1.27 \\
-22.28 & 20.02 \\
0.22 & -0.19 \\
0.33 & -0.29
\end{array}\right] \text {. }
$$

To compare our results with those in [21-23], we give Table 2 in the following. From Table 2 it can be seen that, for this example, our method leads to less conservative results than that obtained in [21] and gives the same results as those obtained in $[22,23]$. 


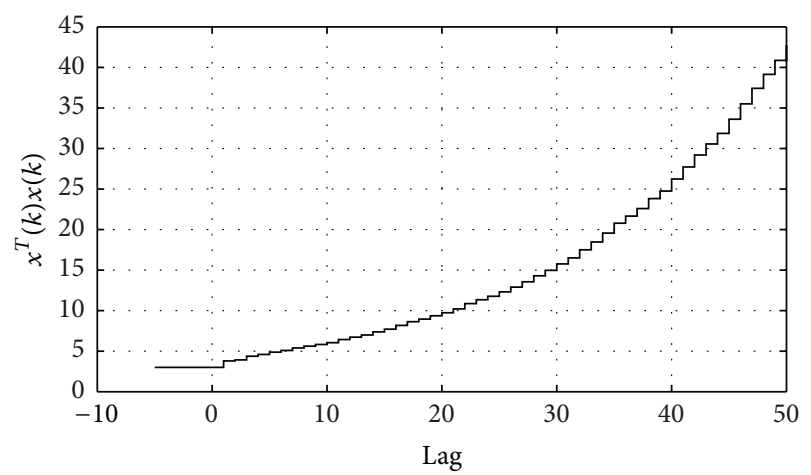

(a)

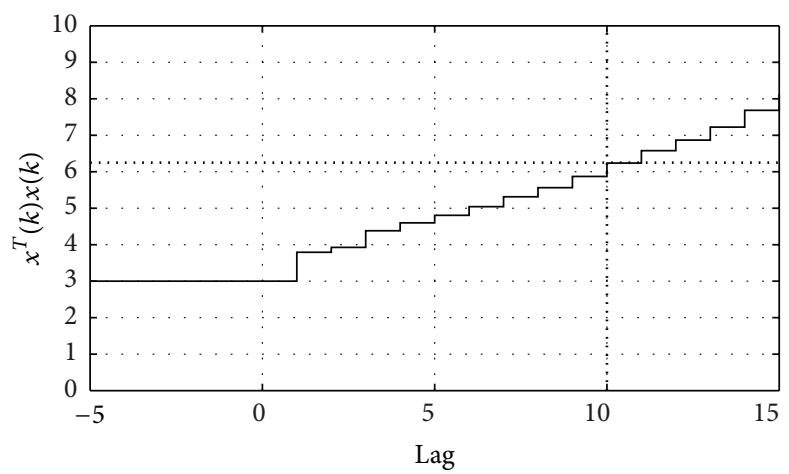

(b)

FIGURE 3: The norm of initial state responses of system (56) for (a) $-10 \leq k \leq 50$ and (b) $-5 \leq k \leq 15$.

In order to verify previous results, we choose the following initial conditions:

$$
\begin{aligned}
& \psi_{\theta \in\{-10,-9, \ldots, 0\}} \\
& =\left[\begin{array}{lll}
\psi(-10) & \cdots & \psi(0)
\end{array}\right] \\
& =\left[\begin{array}{ccccccccccc}
-1 & -1 & -1 & -1 & -1 & -1 & -1 & -1 & -1 & -1 & -1 \\
0 & 1 & 1 & 1 & 1 & 1 & 1 & 1 & 1 & 1 & 1
\end{array}\right]^{T},
\end{aligned}
$$

and we compute the initial state responses of system (60). Obviously, it can be seen that the initial values satisfy the following conditions:

$$
\begin{aligned}
& \sup _{\theta \in\{-10,-9, \ldots, 0\}} \phi^{T}(\theta) \phi(\theta)<\alpha=2.1, \\
& \quad \sup _{\theta \in\{-10,-9, \ldots,-1\}}(\phi(\theta+1)-\phi(\theta))^{T}(\phi(\theta+1)-\phi(\theta)) \\
& \quad \leq \delta=1.1 .
\end{aligned}
$$

Figures 4-5 show the initial state responses $x(k)$ and the norm of initial state responses $x^{T}(k) x(k)$ for the random time-varying delay $2 \leq d(k) \leq 10$. It can be seen that, under the above initial conditions, the state responses of system (60) satisfy the following conditions:

$$
x^{T}(k) x(k)<\beta=80, \quad \forall k \in\{1,2, \ldots, 100\},
$$

which means that the system is finite-time stable with respect to $(2.1,80,100)$.

Example 3. Consider the following discrete-time-delay system with time-varying delay:

$$
\begin{aligned}
x(k+1)= & {\left[\begin{array}{ll}
0.60 & 0.00 \\
0.35 & 0.70
\end{array}\right] x(k) } \\
& +\left[\begin{array}{ll}
0.20 & 0.25 \\
0.25 & 0.15
\end{array}\right] x(k-d(k)),
\end{aligned}
$$

which has been considered in [21,22].

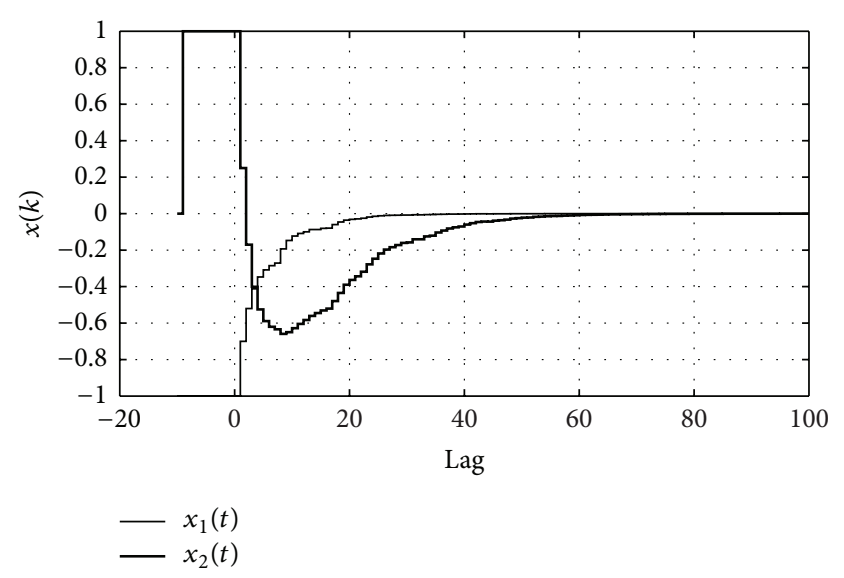

FIGURE 4: Initial state responses of system (60) with time-varying delay $2 \leq d(k) \leq 10$.

Based on Theorem 6, for $d_{m}=1 \leq d(k) \leq 5=d_{M}$, $\alpha=2.1, N=10, \delta=1.1$, and $\gamma=1.44$, we find a feasible solution of (23)-(25) for the smallest eligible value $\beta_{m 1}=299$. Further, the condition of Theorem 1 in [22] is only feasible for $\beta_{m 2}=3017$, while the condition of Theorem 1 in [21] is not feasible for any value of the parameter $\beta$. At the same time, Theorem 1 in [21] gives feasible solution for $\beta_{m 3}=27183$ under the following, more restrictive, conditions: $d_{m}=1 \leq$ $d(k) \leq 3=d_{M}, \alpha=2.1, N=7, \delta=1.1$, and $\gamma=$ 2.98. Therefore, from this example, we can conclude that our method leads to less conservative results than those obtained in $[21,22]$.

In order to verify the stability properties, a computer simulation of the system is performed under the conditions

$$
\begin{aligned}
\phi_{\theta \in\{-5,-4, \ldots, 0\}} & =\left[\begin{array}{llll}
\phi(-5) & \cdots & \phi(0)
\end{array}\right] \\
& =\left[\begin{array}{llllll}
1 & 1 & 1 & 1 & 1 & 1 \\
1 & 1 & 1 & 1 & 1 & 1
\end{array}\right]^{T},
\end{aligned}
$$




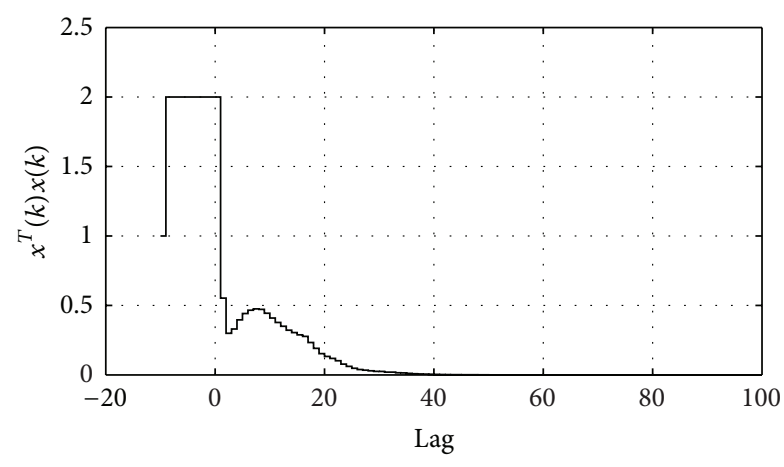

FIGURE 5: The norm of initial state responses of system (60).

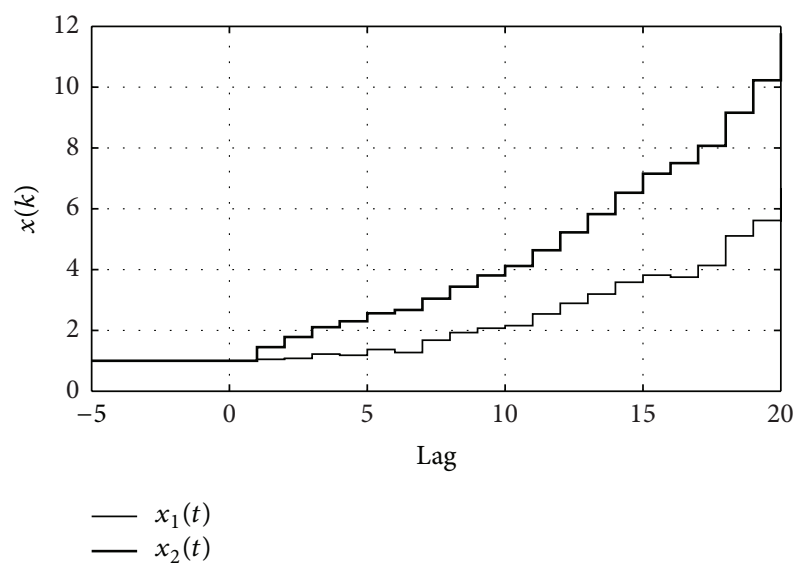

FIGURE 6: Initial state response of system (65) with time-varying delay $1 \leq d(k) \leq 5$.

with the random time-varying delay, $1 \leq d(k) \leq 5$. It is obvious that

$$
\begin{aligned}
& \sup _{\theta \in\{-5,-4, \ldots, 0\}} \phi^{T}(\theta) \phi(\theta) \leq \alpha=2.1, \\
& \sup _{\theta \in\{-5,-4, \ldots,-1\}}(\phi(\theta+1)-\phi(\theta))^{T}(\phi(\theta+1)-\phi(\theta)) \\
& \quad \leq \delta=1.1 .
\end{aligned}
$$

Figures 6-7 show the initial state responses of the above system and the norm of initial state responses. Obviously, the considered system is not asymptotically stable. From Figure 7, the theoretical smallest eligible value of the parameter $\beta, \beta_{m}^{t}$, which is obtained by computer simulation amounts to $\beta_{m}^{t}=23.6$. As the following conditions are satisfied: $\beta_{m 1}=299>\beta_{m}^{t}, \beta_{m 2}=3017>\beta_{m}^{t}$, and $\beta_{m 3}=$ $27183>\beta_{m}^{t}$, we can conclude that system (65) is finite-time stable.

Remark 4. From the previous examples, the following conclusion can be made. In case when the system is not asymptotically stable, the derived results in this paper are less conservative in comparison with those from the existing literature [21-23]. When the system is asymptotically stable, this

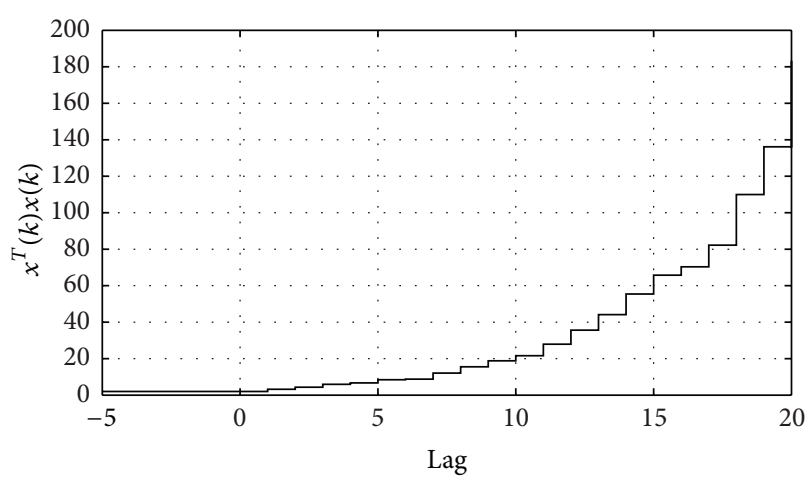

Figure 7: The norm of initial state response of system (65).

paper gives the same results as $[22,23]$ and less conservative results compared to [21].

\section{Conclusion}

In this paper, the problem of finite-time stability of discretetime systems with time-varying delay has been considered. First, in order to obtain less conservative stability criteria, two finite sum inequalities for estimating weighted norms of delayed states are proposed. Then, by using LyapunovKrasovskii-like functionals with power function, new sufficient conditions of finite-time stability are obtained. The stability criteria are expressed in the form of linear matrix inequalities. It was shown that the obtained results are less conservative than some existing ones in the literature.

\section{Conflict of Interests}

The author declares that there is no conflict of interests regarding the publication of this paper.

\section{Acknowledgment}

This work was supported in part by the Ministry of Science and Technological Development of Republic of Serbia under Grant ON174001.

\section{References}

[1] G. V. Kamenkov, "On stability of motion over a finite interval of time," Journal of Applied Mathematics and Mechanics, vol. 17, no. 2, pp. 529-540, 1953.

[2] L. Weiss and E. F. Infante, "Finite time stability under perturbing forces and on product spaces," IEEE Transactions on Automatic Control, vol. 12, no. 1, pp. 54-59, 1967.

[3] F. Amato, M. Ariola, and C. Cosentino, "Finite-time stabilization via dynamic output feedback," Automatica, vol. 42, no. 2, pp. 337-342, 2006.

[4] Y. Shen, "Finite-time control of linear parameter-varying systems with norm-bounded exogenous disturbance," Journal of Control Theory and Applications, vol. 6, no. 2, pp. 184-188, 2008.

[5] E. Moulay and W. Perruquetti, "Finite time stability and stabilization of a class of continuous systems," Journal of 
Mathematical Analysis and Applications, vol. 323, no. 2, pp. 1430-1443, 2006.

[6] L. Zhu, Y. Shen, and C. Li, "Finite-time control of discrete-time systems with time-varying exogenous disturbance," Communications in Nonlinear Science and Numerical Simulation, vol. 14, no. 2, pp. 361-370, 2009.

[7] F. Amato, M. Ariola, and C. Cosentino, "Finite-time control of discrete-time linear systems: analysis and design conditions," Automatica, vol. 46, no. 5, pp. 919-924, 2010.

[8] F. Amato, R. Ambrosino, M. Ariola, and G. de Tommasi, "Input to output finite-time stabilization of discrete-time linear systems," in Proceedings of the 18th IFAC World Congress, pp. 156-161, Milan, Italy, August-September 2011.

[9] J. Cheng, S. Zhong, Q. Zhong, H. Zhu, and Y. Du, "Finite-time boundedness of state estimation for neural networks with timevarying delays," Neurocomputing, vol. 129, no. 10, pp. 257-264, 2014.

[10] S. Wang, T. Shi, M. Zeng, L. Zhang, F. E. Alsaadi, and T. Hayat, "New results on robust finite-time boundedness of uncertain switched neural networks with time-varying delays," Neurocomputing, vol. 151, pp. 522-530, 2015.

[11] N. Yotha and K. Mukdasai, "New delay-dependent robust stability criterion for LPD discrete-time systems with interval time-varying delays," Discrete Dynamics in Nature and Society, vol. 2013, Article ID 929725, 10 pages, 2013.

[12] P.-L. Liu, "Further improvement on delay-range-dependent stability results for linear systems with interval time-varying delays," ISA Transactions, vol. 52, no. 6, pp. 725-729, 2013.

[13] K.-Y. Lian, W.-T. Yang, and P. Liu, "Partitioning technique for relaxed stability criteria of discrete-time systems with interval time-varying delay," Discrete Dynamics in Nature and Society, vol. 2014, Article ID 297324, 6 pages, 2014.

[14] M. P. Lazarević, D. L. Debeljković, Z. L. Nenadić, and S. A. Milinković, "Finite-time stability of delayed systems," IMA Journal of Mathematical Control and Information, vol. 17, no. 2, pp. 101-109, 2000.

[15] F. Gao, Z. Yuan, and F. Yuan, "Finite-time control synthesis of networked control systems with time-varying delays," Advances in Information Sciences and Service Sciences, vol. 3, no. 7, pp. 1-9, 2011.

[16] S. B. Stojanovic, D. L. Debeljkovic, and D. S. Antic, "Finite-time stability and stabilization of linear time-delay systems," Facta Universitatis. Series Automatic Control and Robotics, vol. 11, no. 1, pp. 25-36, 2012.

[17] S. B. Stojanovic, D. L. Debeljkovic, and D. S. Antic, "Robust finite-time stability and stabilization of linear uncertain timedelay systems," Asian Journal of Control, vol. 15, no. 5, pp. 15481554, 2013.

[18] T. Rojsiraphisal and J. Puangmalai, "An improved finite-time stability and stabilization of linear system with constant delay," Mathematical Problems in Engineering, vol. 2014, Article ID 154769, 7 pages, 2014.

[19] G. Zong, R. Wang, W. X. Zheng, and L. Hou, "Finite-time stabilization for a class of switched time-delay systems under asynchronous switching," Applied Mathematics and Computation, vol. 219, no. 11, pp. 5757-5771, 2013.

[20] T. Yu, Y. Zhong, T. Chen, and C. Chen, "Finite-time stabilization of uncertain switched positive linear systems with time-varying delays," Discrete Dynamics in Nature and Society, vol. 2015, Article ID 954782, 9 pages, 2015.
[21] S. B. Stojanovic, D. L. J. Debeljkovic, and N. Dimitrijevic, "Finite-time stability of discrete-time systems with timevarying delay," Chemical Industry and Chemical Engineering Quarterly, vol. 18, no. 4 I, pp. 525-533, 2012.

[22] Z. Zuo, H. Li, and Y. Wang, "New criterion for finite-time stability of linear discrete-time systems with time-varying delay," Journal of the Franklin Institute, vol. 350, no. 9, pp. 27452756, 2013.

[23] Z. Zhang, H. Zhang, B. Zheng, and H. R. Karimi, "Finitetime stability analysis and stabilization for linear discretetime system with time-varying delay," Journal of the Franklin Institute, vol. 351, no. 6, pp. 3457-3476, 2014. 


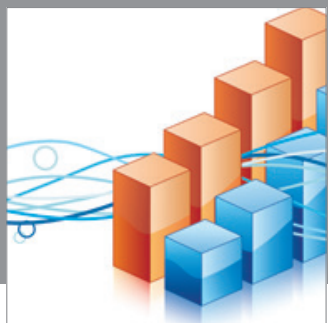

Advances in

Operations Research

mansans

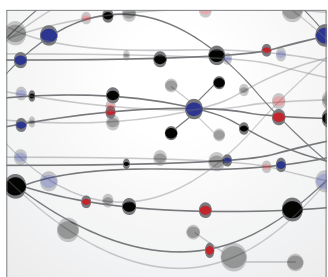

The Scientific World Journal
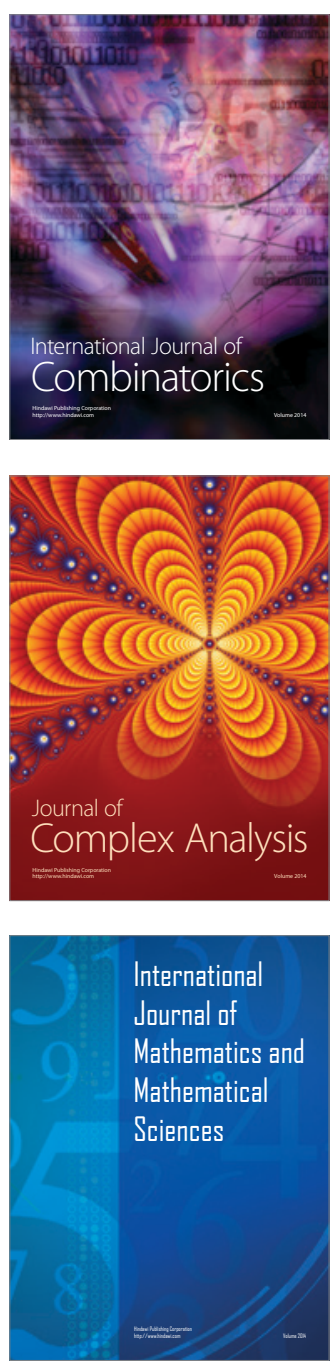
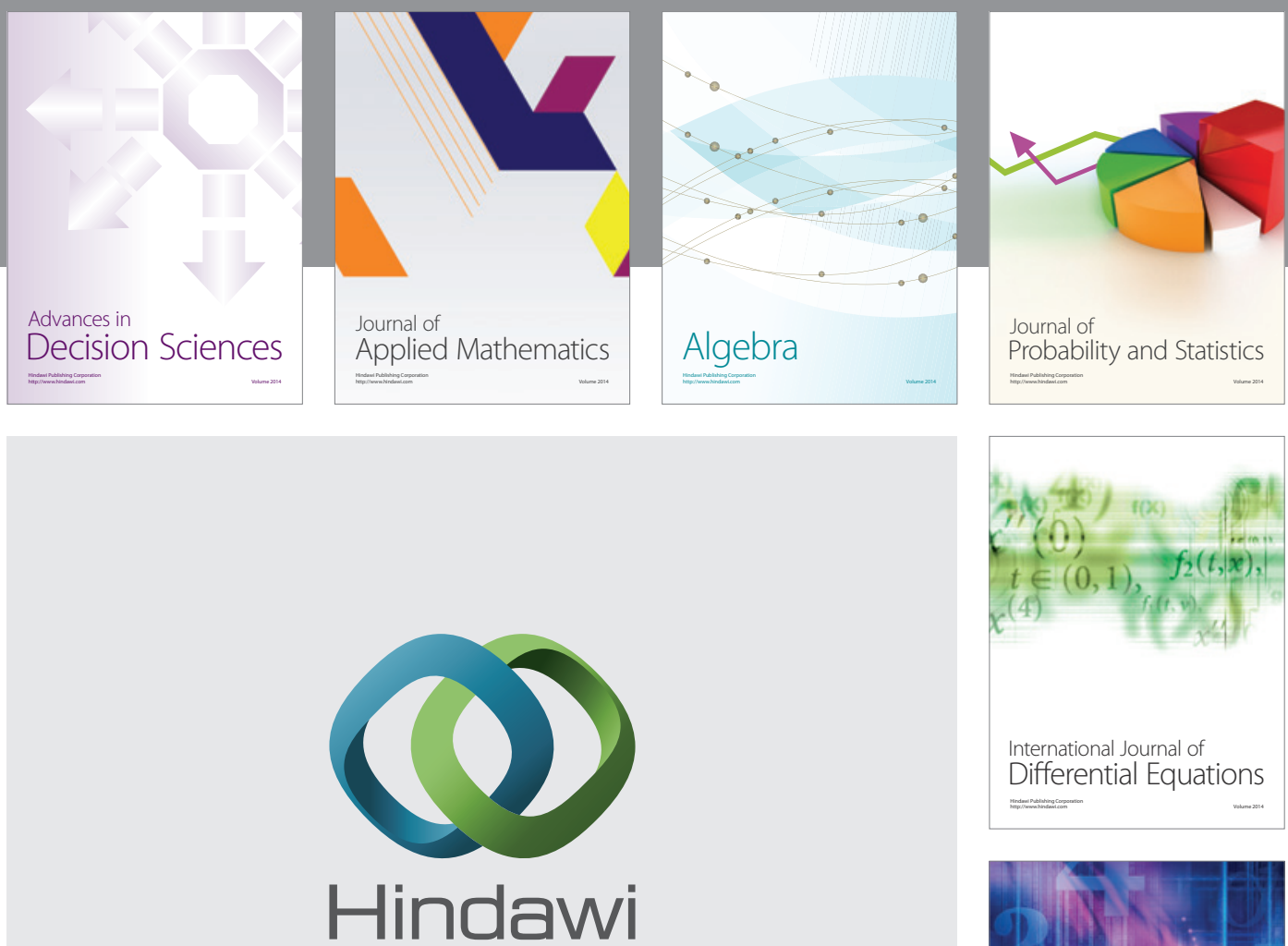

Submit your manuscripts at http://www.hindawi.com
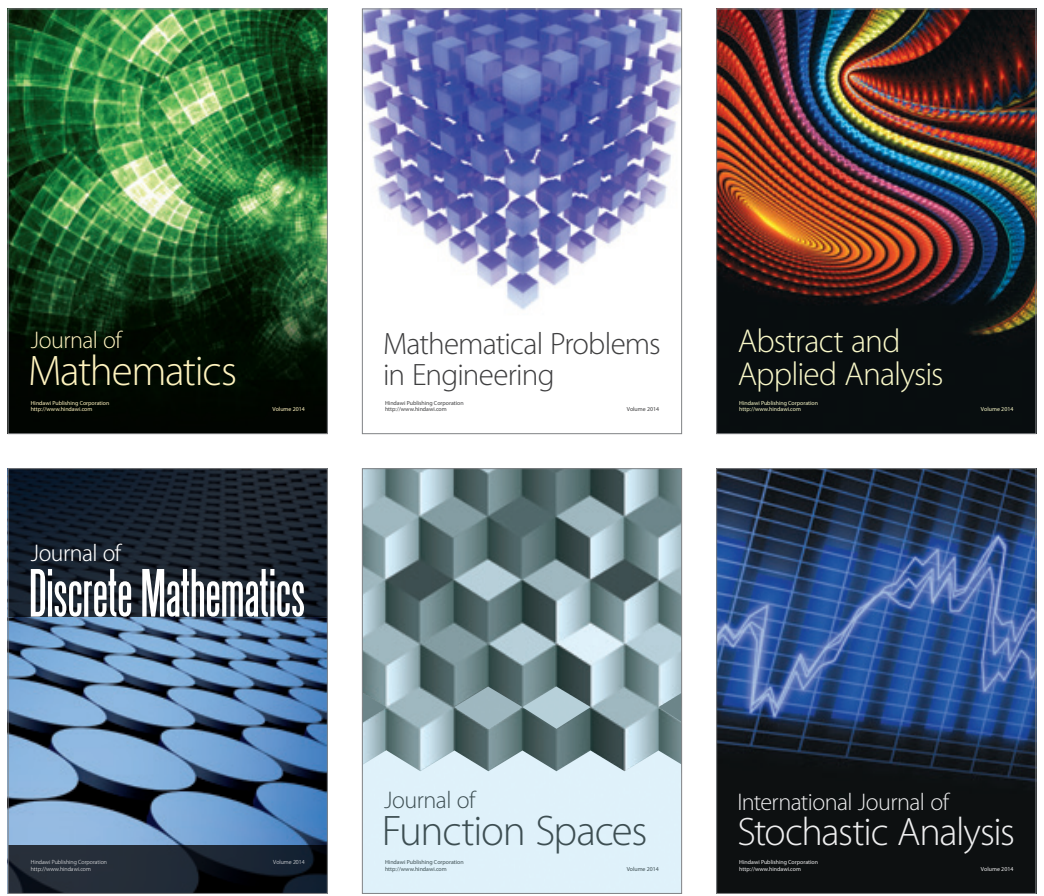

Journal of

Function Spaces

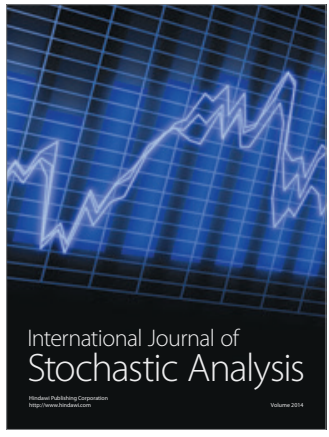

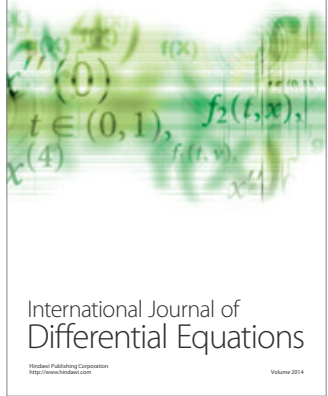
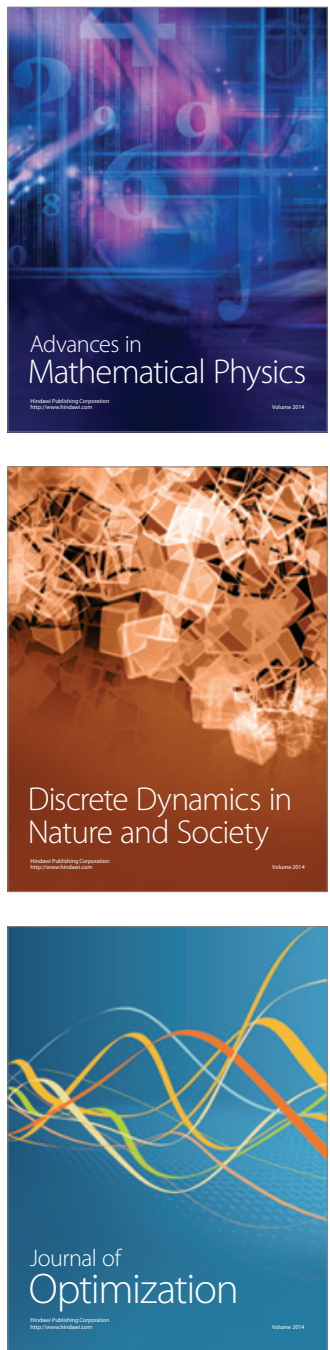